\title{
Treatment and Outcome of 341 Papillary Breast Lesions
}

\author{
Peixian Chen ${ }^{1} \cdot$ Dan Zhou ${ }^{2} \cdot$ Chuan Wang ${ }^{3} \cdot$ Guolin $\mathrm{Ye}^{2} \cdot{\text { Ruilin } \text { Pan }^{2} \cdot \text { Lewei Zhu }}^{2}$
}

Published online: 17 June 2019

(C) The Author(s) 2019

\begin{abstract}
Background Papillary breast lesions constitute a pathological heterogeneous group and display diverse clinical and imaging features. This study was conducted to analyze the upgrade rate of intraductal papilloma diagnosed on core needle biopsy and to assess the possible risk factors associated with upgrade to higher-risk lesions. We also examined the long-term outcomes in patients who received resection of the papillary lesions.

Materials and methods The clinical and pathology records of 324 female patients who were diagnosed with papillary lesions based on core needle biopsy (CNB) from February 2010 to October 2016 at our institution were retrospectively analyzed. Patients were grouped by initial diagnosis into two groups (papilloma with or without atypia) and followed-up for long-term outcomes. For the upgrade to higher-risk lesions after excision, upgraded lesions were compared with benign papillomas for the collected variables.

Results A total of 341 lesions were included for final analysis, and all were available for follow-up. Papillomas with or without atypia diagnosed by CNB were found in 9 and 332 lesions, respectively. Papillomas without atypia on CNB were treated by open excision $(n=265)$ or vacuum-assisted biopsy (VAB) $(n=67)$, which yielded similar event-free rate $(p=0.19)$. The upgrade rate of this group to higher-risk lesions was $9.9 \%$. Peripheral $(p=0.011)$ lesions in postmenopausal $(p=0.001)$ or older $(p=0.001)$ patients with papillomas without atypia based on CNB showed significantly higher upgrade rates. Papillomas with atypia on CNB were all managed by open excision, and concurrent malignancy was found in two lesions.

Conclusion In conclusion, our results support benign papillary lesions based on CNB require further treatment. Peripheral lesions occurring in older or postmenopausal women are at higher risk for upgrade.
\end{abstract}

The authors Peixian Chen and Dan Zhou contributed equally to this work.

Guolin Ye

13902816950@139.com

1 Sun Yat-Sen Memorial Hospital, Sun Yat-Sen University, 107 Yanjiang West Road, Guangzhou 510120, People's Republic of China

2 Department of Breast Surgery, The First People's Hospital of Foshan, \#81, North Lingnan Avenue, Chancheng, Foshan, Guangdong, People's Republic of China
3 The First People's Hospital of Foshan, \#81, North Lingnan Avenue, Chancheng, Foshan, Guangdong, People's Republic of China 


\section{Introduction}

Papillary lesions of the breast include a broad spectrum of proliferative diseases, ranging from benign to malignant lesions [1]. In clinical practice, papillary lesions may appear as spontaneous nipple discharge, detection on mammography or, infrequently, a palpable mass [2]. Nevertheless, the clinical presentation or age of the patients is unreliable to differentiate between benign, atypical or malignant lesions [3].

The diagnosis and treatment of papillary lesions is greatly challenging. Papillomas are usually detected by mammography and ultrasound (US) and subsequently diagnosed by fine needle aspiration (FNA), core needle biopsy (CNB) and vacuum-assisted biopsy (VAB) [4]. Although imaging-guided CNB is a reliable method for the diagnosis of breast lesions, papillary lesions identified at percutaneous biopsy can be difficult to interpret histologically [5]. Also, limited material or sampling error at CNB may result in missing the presence of atypia or malignancy within or adjacent to the lesion [6].

Surgical excision is typically performed for papillary lesions following CNB and generally recommended to rule out concurrent malignancy when a diagnosis of papilloma with atypia is yielded on CNB [7]. For papillomas without atypia, however, recommendations for excision versus observation are conflicting. Some prefer surgical excision because $17-24 \%$ of lesions can be underestimated by CNB [8] while others support follow-up with repeated imaging owing to low rates of malignancy of benign papillomas [9]. Although excisional biopsy is considered gold standard, for treating papillary lesions, VAB has been proved to be effective in removing benign papillomas instead of excisional biopsy [4].

The primary aim of this study was to evaluate the rate of upgrade to higher-risk lesions of papillomas diagnosed on CNB. We also sought to analyze the possible risk factors for upgrade. The secondary aim was to determine the longterm outcomes in patients who had resection of the papillary lesions.

\section{Patients and methods}

A total of 429 patient records were drawn from the pathology computer database at our institution for women who underwent a biopsy between 2010 and 2016, using a keyword-based search with words including "papilloma," and "papillary." All duplicated records were removed $(n=70)$. Patients were excluded if breast cancer was diagnosed on initial core needle biopsy (CNB) $(n=17)$ or initial excision $(n=12)$. Patients who were followed-up less than 6 months $(n=1)$ and those who had a history of breast cancer $(n=4)$ were also removed. Patients in whom no papillary lesion or papilloma was found on excision were also excluded $(n=1)$. The study cohort comprised 324 patients and yielded 341 lesions for final analysis (Fig. 1). Two groups were defined based on CNB diagnosis: papilloma without atypia and papilloma with atypia.

$\mathrm{CNB}$ was performed using a $14 \mathrm{G}$ needle. All patients who had a CNB underwent excision. VAB was performed using an 8 G needle (Mammotome ${ }^{\circledR}$ Biopsy System $300 \mathrm{E}$ Business Way, Cincinnati, OH).

The slides from the surgical pathology were re-evaluated for this study. According to the World Health Organization (WHO) Classification of Breast Tumors, papillary lesions of the breast can be classified as intraductal papilloma, intraductal papilloma with atypical ductal hyperplasia (ADH) or with ductal carcinoma in situ (DCIS) or with lobular neoplasia (LN), papillary DCIS, encapsulated papillary carcinoma and solid papillary carcinoma. In this study, "atypia" within a papilloma was defined as the presence of ADH or atypical lobular hyperplasia (ALH). Lesion locality was defined according to ultrasound findings. A lesion within $2 \mathrm{~cm}$ of the nipple was defined as a central lesion, and any location further away was defined as a peripheral location.

Patients received both clinical breast examination and routine breast ultrasound examination on follow-up. The occurrence of events during the follow-up period (until the 31st October 2018) was evaluated using the Kaplan-Meier method. Lesions that occurred during the follow-up were classified as papilloma without atypia, papilloma with atypia and breast cancer. All statistical tests were performed at an alpha level of 0.05. The Stata 12.0 software was used for all analysis.

\section{Results}

\section{Patient characteristics}

A total of 341 papillary lesions were diagnosed by CNB. The mean age of the patients was 43.5 years (median: 43 years; range: 18-79 years), and most of the patients were premenopausal $(n=266,82.1 \%)$. Asymptomatic lesions which were detected by imaging accounted for $40.8 \%$ ( $n=139)$. Overall, 140 lesions $(41.1 \%)$ occurred as palpable tumors and 86 lesions(25.2\%)presented with abnormal nipple discharge. Nipple discharge appeared in 24 palpable lesions (7.0\%).

CNB was mostly indicated according to radiologic findings. All the lesions diagnosed as papilloma without atypia ( $n=332)$ on CNB presented with abnormal nipple discharge, of which 34 could be found on ductoscopy, and 


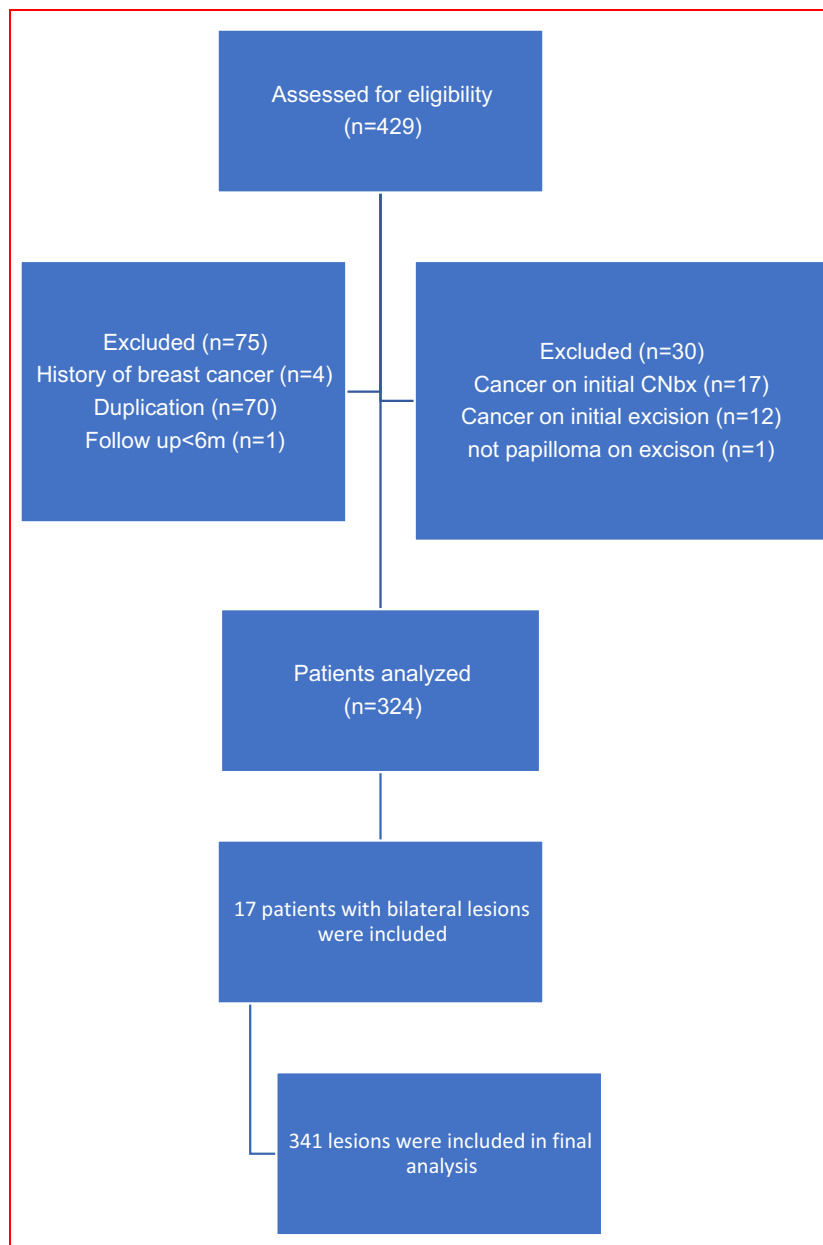

Fig. 1 Flowchart of patient inclusion

137 were palpable. Lesions diagnosed as papilloma with atypia $(n=9)$ on CNB did not present with a nipple discharge, and only three were palpable. There were 133 and 6 asymptomatic lesions in those without or with atypia, respectively.

\section{Treatment}

Surgical excision including open biopsy (OB) or VAB following $\mathrm{CNB}$ evaluation was performed for all lesions. (Fig. 2) For papillomas without atypia, OB or VAB was performed in 265 and 67 lesions, respectively. Those excised using OB turned out to be pure papillomas in 238 lesions, concurrent malignancy in 6 lesions and papillomas with atypia in 21 lesions. Those removed by VAB were found to be benign papillomas in 61 lesions, concurrent malignancy in one lesion and papilloma with atypia in 5 lesions. The lesion diagnosed as malignancy by VAB was subsequently excised using $\mathrm{OB}$, and the pathological finding was consistent with the result of VAB. Invasive carcinoma was not found in any lesion. All of the 9 lesions that were considered papillomas with atypia based on CNB were removed using $\mathrm{OB}$, of which the pathological findings of the surgical specimen were three papillomas, two malignancies and four atypical papillomas. Patients underwent mastectomy or lumpectomy when malignancy was confirmed by OB. Patients received chemo-preventive therapy, either tamoxifene or tomirefene, when atypia was diagnosed.

\section{Re-classification}

Table 1 summarizes the results of comparison among clinical variables for upgrade rates among papillomas without atypia on CNB. The upgrade rate of this group to higher-risk lesions (cancer or atypia) was 9.9\% (33 of 332 lesions). Lesions in older or postmenopausal patients showed a significantly higher rate of upgrade than those in younger $(p=0.001)$ or premenopausal $(p=0.001)$ patients. Peripheral lesions were more likely to upgrade than central lesions $(p=0.011)$. No significant difference as to surgical history of benign breast tumor, family history of breast cancer, nipple discharge or palpable disease in upgrade rate was found.

The mean age of the nine patients whose lesions [solid papillary carcinoma $(n=4)$; ductal carcinoma in situ $(n=5)$ ] were reclassified as malignancy was 50.4 years (median: 47 year; range: 39-63 year). Five of these patients were premenopausal, and one had a family history of breast cancer. One of these patients presented with abnormal nipple discharge, and five had a palpable mass.

\section{Follow-up}

Follow-up data were available and analyzed for all lesions that were treated either by VAB or OB after CNB. All patients were alive when follow-up was censored on October 31, 2018. The median follow-up time was 44.5 months and ranged from 16 months to 101 months. Kaplan-Meier analysis was performed with the time of surgical excision as the index point, and patients were censored according to events occurring during the followup period, including recurrence of the papilloma $(n=9$; $60.0 \%)$, detection of another high-risk lesion $(n=1 ; 6.7 \%)$, or malignancy $(n=5 ; 33.3 \%)$. In 15 cases, a subsequent lesion was found and was considered as an event in the Kaplan-Meier curve. (Fig. 2).

Malignancy developed in five papillomas without atypia based on CNB during follow-up: two lesions were diagnosed as papillomas with atypia on open biopsy, and three lesions were diagnosed as benign papillomas on VAB $(n=1)$ and open biopsy $(n=2)$. One of the papillomas recurred and developed malignancy at follow-up period of 8 months and 33 months, respectively. One atypical 


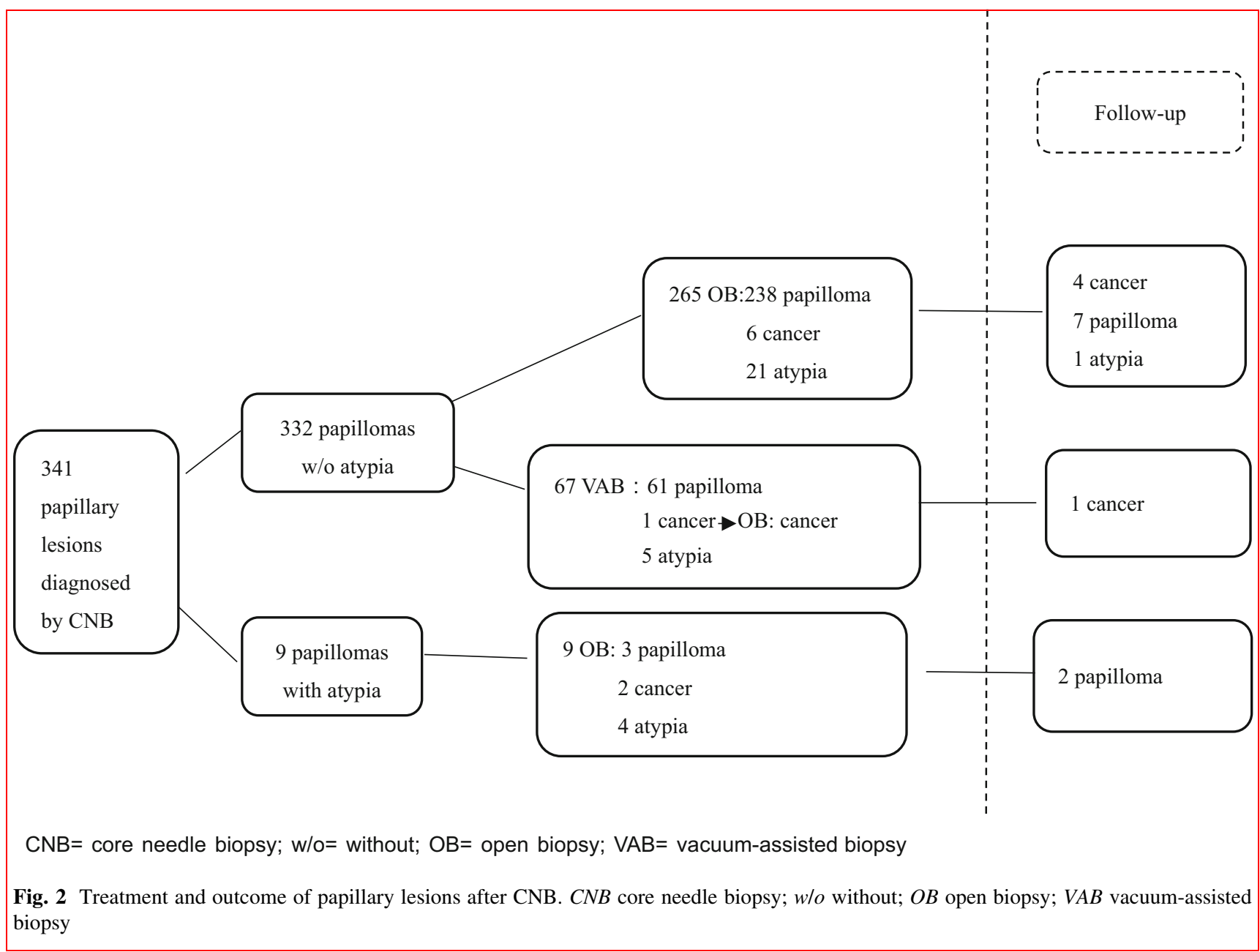

papilloma and six benign papillomas occurred in another seven papillomas without atypia both on CNB and on open biopsy. Among papillomas with atypia on $\mathrm{CNB}$, benign papillomas occurred in two lesions which were diagnosed with atypia on open excision. (Fig. 2).

The overall event-free rate (EFS) for all lesions was $95.9 \%$. No significant differences were found in papillomas without atypia treated using $\mathrm{OB}$ or $\mathrm{VAB}$ after $\mathrm{CNB}$ $(p=0.19)$ (Fig. 3).

\section{Discussion}

The preferred management following the diagnosis of papillary lesions of the breast based on CNB has been contentious. Surgical excision of papillary lesions is widely recommended especially for those with atypia due to their high association with malignancy. However, the case is not the same with the treatment of papillary lesions without atypia. Discrepancy as to the upgrade rate from a papillary lesion without atypia to a cancer diagnosis has been noted, ranging from 2 to $33 \%$ [10]. The reason for this discrepancy has been intensively investigated. Factors such as lesion size, nipple discharge and palpable disease, have been reported to be associated with upgrading; however, results are not consistent. We investigated the upgrade rate of papillary lesions from their initial CNB diagnosis, the effect of managing papillary lesion without atypia using $\mathrm{VAB}$ and the likelihood of occurrence of papilloma or cancer on long-term follow-up for every patient.

Our study looked at a cohort of 324 patients with 341 papillary lesions with or without atypia diagnosed by CNB. Our study showed a nearly $10 \%(33 / 332,9.9 \%)$ of papillomas without atypia which were underestimated by CNB would, on excision, have atypia or malignancy while Wyss et al. [4] noted $8.7 \%$ lesions were reclassified from a benign papillary lesion to a higher-grade lesion or malignancy. We also found that two out of nine papillomas with atypia (22.2\%) on CNB were upgraded to malignancy. A meta-analysis suggested that more than $1 / 3$ of the papillary lesions with atypia on $\mathrm{CNB}$ were upgraded to cancer compared with that only $7 \%$ of those without atypia were 
Table 1 Clinical variables and final diagnosis of papillomas without atypia on CNB

\begin{tabular}{|c|c|c|c|c|c|}
\hline & & \multicolumn{2}{|l|}{ Final diagnosis } & \multirow{2}{*}{$\begin{array}{l}\text { Percentage upgraded to higher- } \\
\text { risk lesions }(\%)\end{array}$} & \multirow[t]{2}{*}{$p$ value } \\
\hline & & $\begin{array}{l}\text { Papilloma without } \\
\text { atypia }\end{array}$ & $\begin{array}{l}\text { Papilloma with atypia or } \\
\text { malignancy }\end{array}$ & & \\
\hline Age(yrs) & Mean \pm SD & $43.0 \pm 0.6$ & $50.0 \pm 2.0$ & - & $0.001 *$ \\
\hline \multirow[t]{2}{*}{ Menstruation status N (\%) } & Premenopause & $251(92.6)$ & $20(7.4)$ & 7.4 & \multirow[t]{2}{*}{$0.001 *$} \\
\hline & Postmenopause & $48(78.7)$ & $13(21.3)$ & 21.3 & \\
\hline \multirow{2}{*}{$\begin{array}{l}\text { History of benign Breast } \\
\text { tumor } \mathrm{N}(\%)\end{array}$} & Yes & $31(96.9)$ & $1(3.1)$ & 3.1 & \multirow[t]{2}{*}{0.23} \\
\hline & No & $268(89.3)$ & $32(10.7)$ & 10.7 & \\
\hline \multirow{2}{*}{$\begin{array}{l}\text { Family history of breast } \\
\text { cancer } N(\%)\end{array}$} & Yes & $1(50.0)$ & $1(50.0)$ & 50.0 & \multirow[t]{2}{*}{0.19} \\
\hline & No & $298(90.3)$ & $32(9.7)$ & 9.7 & \\
\hline \multirow[t]{2}{*}{ Nipple discharge $\mathrm{N}(\%)$} & Yes & $77(89.5)$ & $9(10.5)$ & 10.5 & \multirow[t]{2}{*}{0.85} \\
\hline & No & $222(90.2)$ & $24(9.8)$ & 9.8 & \\
\hline \multirow[t]{2}{*}{ Palpable lesion N (\%) } & Yes & $121(88.3)$ & $16(11.7)$ & 11.7 & \multirow[t]{2}{*}{0.38} \\
\hline & No & $178(91.3)$ & $17(8.7)$ & 8.7 & \\
\hline \multirow{2}{*}{$\begin{array}{l}\text { Lesion distance from the } \\
\text { nipple } \mathrm{N}(\%)\end{array}$} & Central & $142(94.7)$ & $8(5.3)$ & 5.3 & \multirow[t]{2}{*}{$0.011 *$} \\
\hline & Peripheral & $157(82.3)$ & 25 (13.7) & 13.7 & \\
\hline
\end{tabular}

$* p<0.05$

upgraded to cancer [7]. Sidrah Khan et al. [11] also found that concomitant invasive or ductal in situ cancer appeared in a greater than $30 \%$ of cases on excision of which the initial CNB diagnosis was papilloma with atypia while the likelihood of cancer was much lower in those without atypia on CNB. Since our study yielded similar results to that in the literature, we agree that papillomas diagnosed by CNB should be considered for further treatment.

Research has been carried out to identify the possible risk factors for malignant papillomas, including patient age on diagnosis, lesion location, the presence of nipple discharge, tumor size and radiologic findings. The impact of age was controversial that some showed a higher underestimation rate in patients age under 50 years, whereas others concluded differently $[6,12]$. Peripherally located [3] and larger papillomas [6, 12, 13] are also more likely to be underestimated than centrally located and smaller papillomas. In our study, we found a significantly higher upgrade rate of lesions located peripherally in older or postmenopausal patients, which is consistent with the literature. We failed to demonstrate that the presence of a nipple discharge or palpable disease correlated with upgrade.

$\mathrm{VAB}$ is considered more accurate in terms of both sensitivity and specificity than CNB. Some have proposed conservative management of papillary lesions of the breast by VAB instead of by open biopsy. Chang et al. [13] performed US-guided VAB to remove lesions, none of which were reclassified as high risk. Wyss et al. also demonstrated that VAB of papillomas did not underestimate the pathology when compared to excision biopsy ${ }^{4}$.

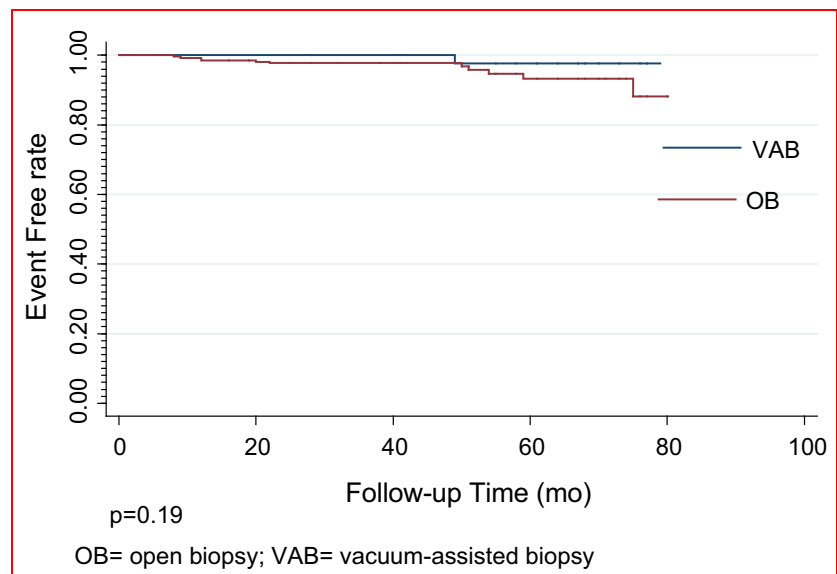

Fig. 3 Event-free rate of patients with papillomas w/o atypia treated using $\mathrm{OB}$ or VAB. $O B$ open biopsy; $V A B$ vacuum-assisted biopsy

Given that $\mathrm{VAB}$ is less invasive, less expensive and quicker to perform, it may be preferred over open biopsy, which is the current gold standard in treating papillary lesions ${ }^{4}$. At our institution, we are experienced in using $\mathrm{VAB}$ to treat selective small benign breast tumors $(\leq 2 \mathrm{~cm})$. In this study, lesions without atypia based on $\mathrm{CNB}$ which were excised by VAB did not seem more likely to recur or develop malignancy. Therefore, papillary without atypia diagnosed by CNB can be managed by $\mathrm{VAB}$. However, further research is required to assess the safety of this approach. 
We followed all patients for a median period of 44.5 months (range 16-101 months) and assessed the likelihood of papilloma recurrence and higher-risk lesions on long-term follow-up. The overall event-free survival (EFS) was $96.1 \%$ for papillomas without atypia on CNB, compared to $77.8 \%$ for papillomas with atypia. This indicates that the finding of atypia with a papillary lesion tends to be at a higher risk for recurrence or developing upgraded lesions during long-term follow-up, although the group of papillomas with atypia was too small for meaningful analysis.

Our study had some limitations. First, we performed a retrospective study based on a single breast center. However, this study represents one of the largest cohorts of its kind published to date since it is difficult to establish a large cohort of patients with such a rare diagnosis. Second, the median follow-up of $44.5 \mathrm{mo}$ is relatively short and some recurrence or even malignancy may not be detectable at this time. Third, the group of patients diagnosed with papillomas with atypia on CNB is too small to allow further analysis of intergroup difference.

\section{Conclusion}

In conclusion, our results support further treatment for papillomas diagnosed by $\mathrm{CNB}$, especially for peripherally lesions and those occurring in older or postmenopausal women. In our experience, VAB is an accurate method for the diagnosis and treatment for papilloma, and therefore we propose that benign papillary lesions based on CNB can be excised by VAB or OB. However, further studies based on larger cohort with longer follow-up period are essential.

\section{Compliance with ethical standards}

Conflict of interest The authors declare that they have no conflict of interest.

Open Access This article is distributed under the terms of the Creative Commons Attribution 4.0 International License (http://crea tivecommons.org/licenses/by/4.0/), which permits unrestricted use, distribution, and reproduction in any medium, provided you give appropriate credit to the original author(s) and the source, provide a link to the Creative Commons license, and indicate if changes were made.

\section{References}

1. Rizzo M, Linebarger J, Lowe MC et al (2012) Management of papillary breast lesions diagnosed on core-needle biopsy: clinical pathologic and radiologic analysis of 276 cases with surgical follow-up. J Am Coll Surg 214:280-287

2. Cabioglu N, Hunt KK, Singletary SE et al (2003) Surgical decision making and factors determining a diagnosis of breast carcinoma in women presenting with nipple discharge. Journal of the American College of Surgeons 196:354-364

3. Kil WH, Cho EY, Kim JH et al (2008) Is surgical excision necessary in benign papillary lesions initially diagnosed at core biopsy? Breast 17:258-262

4. Wyss P, Varga Z, Rossle M et al (2014) Papillary lesions of the breast: outcomes of 156 patients managed without excisional biopsy. Breast J 20:394-401

5. Carder PJ, Khan T, Burrows P et al (2008) Large volume "mammotome" biopsy may reduce the need for diagnostic surgery in papillary lesions of the breast. J Clin Pathol 61:928-933

6. Youk JH, Kim EK, Kwak JY et al (2011) Benign papilloma without atypia diagnosed at US-guided 14-gauge core-needle biopsy: clinical and US features predictive of upgrade to malignancy. Radiology 258:81-88

7. Wen X, Cheng W (2013) Nonmalignant breast papillary lesions at core-needle biopsy: a meta-analysis of underestimation and influencing factors. Ann Surg Oncol 20:94-101

8. Cyr AE, Novack D, Trinkaus K et al (2011) Are we overtreating papillomas diagnosed on core needle biopsy? Ann Surg Oncol 18:946-951

9. Sohn V, Keylock J, Arthurs Z et al (2007) Breast papillomas in the era of percutaneous needle biopsy. Ann Surg Oncol 14:2979-2984

10. Menes TS, Rosenberg R, Balch S et al (2014) Upgrade of highrisk breast lesions detected on mammography in the breast cancer surveillance consortium. Am J Surg 207:24-31

11. Khan S, Diaz A, Archer KJ et al (2018) Papillary lesions of the breast: to excise or observe? Breast J 24:350-355

12. Plantade R, Gerard F, Hammou JC (2006) Management of non malignant papillary lesions diagnosed on percutaneous biopsy. J Radiol 87:299-305

13. Chang JM, Moon WK, Cho N et al (2011) Management of ultrasonographically detected benign papillomas of the breast at core needle biopsy. AJR Am J Roentgenol 196:723-729

Publisher's Note Springer Nature remains neutral with regard to jurisdictional claims in published maps and institutional affiliations. 